\title{
Vibration Damping Measurement on Car Windshields
}

\author{
Balázs Vehovszky*, István Horváth², Karl Slenczka², Martin Schuster², Tamás Jakubík \\ ${ }^{1}$ Department of Whole Vehicle Engineering, Audi Hungaria Faculty of Automotive Engineering, Széchenyi István University, \\ H-9026 Győr, Egyetem tér 1., Hungary \\ 2 Department of Whole Vehicle Development, Audi Hungária Zrt., H-9027 Győr, Audi Hungária út 1., Hungary \\ *Corresponding author, e-mail: vehovszky.balazs@sze.hu
}

Received: 05 October 2017, Accepted: 03 October 2018, Published online: 13 November 2018

\begin{abstract}
Knowledge of the damping properties of a windshield is a fundamental element of the acoustical characterization of a car. The measuring method of damping for a windshield is presented in the paper. The damping loss factor - as a basic measure of mechanical damping - was determined experimentally by two means: the reverberation time from impact hammer testing as well as the modal behavior from 3D laser scanning vibrometer measurements. The results proved that the modal shapes have a fundamental effect on the measured damping values.
\end{abstract}

\section{Keywords}

windshield vibration, modal analysis, damping loss factor, laser scanning vibrometer

\section{Introduction}

Since a windshield has great impact on the acoustic properties of a vehicle, the identification of its vibration properties is essential for the acoustic design of a car's passenger compartment. In this work, the modal behavior as well as the damping properties of a windshield were examined experimentally. The derivation of these properties by measuring on simplified models (e.g. prismatic rod) $[1,2]$ or via finite element modelling [3] is a rather common procedure for simple materials. However, the examination of real windshields - even free-free state (i.e. hanged on elastic ropes) or in a mounted state (i.e. mounted in the chassis) - can provide further important, case-specific information. The aim of present work is to develop the optimal method for the acoustic characterization of a car windshield, and to experimentally determine those vibration properties, which can be useful in executing more accurate acoustic simulations or design.

\section{Measure of vibration damping}

Vibration damping properties can be divided into two major groups: material damping and structural damping. The former can be considered as a pure material property, which can be determined by standardized methods, while the latter one depends mostly on such geometrical and structural features as the bindings, macrostructure or mounted elements - beside the properties of composition materials, obviously. From this point of view, a windshield
- as a geometrically and structurally complex part with specific manufacturing technology - should be treated as a structure rather than a simple "set of materials" and thus, it should be investigated as a whole. Data reached in such way could complement the "classical" data obtained from simplified test specimen-based testing.

For the determination of structural damping there are numerous methods in the literature [4]. The elbowroom of investigation is further expanded by the high number of index numbers for the characterization of damping (e.g. logarithmic decrement, half power bandwidth, damping ratio, specific damping capacity, damping loss factor, loss angle etc.). For windshield characterization, the damping loss factor (DLF) was chosen as the most suitable measure of damping because of such parameters, as high damping value and multi degree of freedom - beside the relative ease of determining DLF.

The damping loss factor $(\eta)$ is the most comprehensive value for damping representation [4]. In general it is specified as the ratio of the imaginary and the real parts of the complex modulus:

$\eta=\frac{E^{\prime \prime}}{E^{\prime}}=\operatorname{tg} \varphi$

where $E^{\prime \prime}$ is the loss modulus, $E^{\prime}$ is the storage modulus and $\varphi$ is the loss angle. It can be interpreted as the energy loss 
per radian, normalized by the whole energy of the vibration. Damping loss factor can be expressed from other measures of vibration damping. In this paper the expression from logarithmic decrement was further improved to reach the correlation between DLF and reverberation time as follows [5]:

$\eta=\frac{\ln 10^{6}}{\omega \cdot T_{60}}=\frac{2.2}{f \cdot T_{60}}$

where $f$ is the frequency and $T_{60}$ is the reverberation time. The latter one means the period of time needed for the vibration energy to decrease to the one-millionth part of its original value. This corresponds to a $60 \mathrm{~dB}$ drop while the vibration amplitude decreases one-thousandth times. Supposing exponential decay $T_{60}$ can be expressed via linear extrapolation from the times required for a $30 \mathrm{~dB}$ or $20 \mathrm{~dB}$ decay (usually designated as $T_{30}$ and $T_{20}$, respectively).

\section{Experiments}

Two types of windshields were used in the experiments: a "normal" and an "acoustic" one (noted with $\mathrm{N}$ and A, respectively). The latter one has higher damping value, with better acoustic properties. Both types have the following sandwich structure: Glass layer - Normal/Acoustic polymer layer - Glass layer with the thicknesses of $2.1 \mathrm{~mm}$ $-0.76 \mathrm{~mm}-1.6 \mathrm{~mm}$, respectively. The local damping loss properties were investigated via impact excitation and local acceleration measurement. The results of this measurement were complemented and verified with the findings of vibration shape analysis.

\subsection{Impact measurements}

The basic principle of DLF measurement is exciting the system by an impulse (e.g. with an impulse-hammer) and measuring the response at the same point (e.g. the acceleration). Knowing the exact excitation and the response, the frequency response function (FRF) and the impulse-response can be calculated. By filtering the response to the required frequency band and plotting the amplitude-decay, $T_{60}$ can be determined. The amplitude decay curve could be enhanced by the Schröder-integration of the sign, which results in a smooth, well evaluable curve [6].

Raw force (excitation) and acceleration (response) data were processed in the Test.Lab program of LMS using the Reverberation time module. Measurements were thereafter processed in an in-house MATLAB code, offering more flexibility and transparency for the calculations.
The comparison of the two methods is unfortunately beyond the limits of the present paper and hence is omitted from this document. A diagram of the data processing flow is plotted in Fig. 1. The excitation with impact hammer (type $\mathrm{PCB} / 086 \mathrm{C} 03$ ) was applied near to the location where the piezo accelerometer (Dytran 3035B2G) was placed.

The whole surface of the investigated windshield was mapped by impact measurements trough 50 measured points (with 3 measurements at each - see Fig. 2). DLF maps for the whole surface of the windshields were then constructed for $1 / 3$ octave frequency bands.

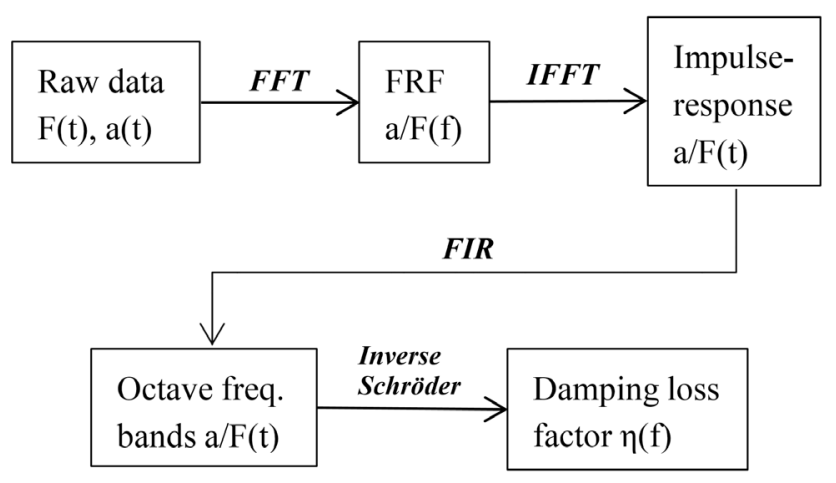

Fig. 1 Block-diagram of data processing

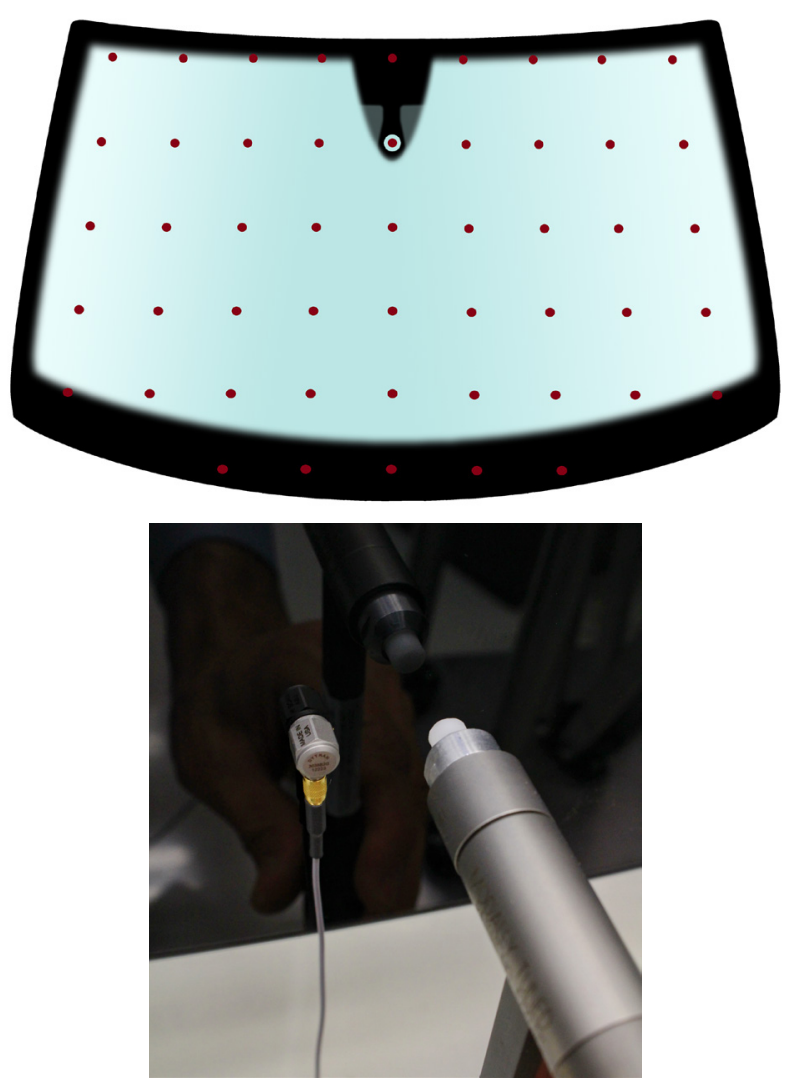

Fig. 2 Measured points on the windshield (up) and measurement with impact hammer and piezo accelerometer (down) 


\subsection{Vibration shape analysis}

Beside the damping loss factor measurements, the modal behavior of a free-free hanged windshield was investigated beside stationary excitation by an electrodynamic shaker (TIRA/S51110) and vibration measurement by a laser-scanning-vibrometer (PSV-400). The major aim of this investigation was to interpret the results of DLF measurements. The experimental setup is illustrated in Fig. 3. The analysis was executed in a frequency range up to $1 \mathrm{kHz}$. As a result, the transfer functions were obtained at each measured points. Using this data, vibration shapes could be visualized. Further information was derived from the average velocity of all measured points, examined as a function of excitation frequency.

\section{Results and discussion}

\subsection{Normal and acoustical windshield}

The difference between the two types of windshields can be clearly understood according to the result plotted in Fig. 4. Note that all results are indicated in normalized form. It can be observed that the modal character (i.e. the resonance peaks) of acoustic windshield disappears at significantly lower frequency than that of the normal one ( $f_{1}$ for the acoustic windshield in comparison to $f_{2} \approx 4 f_{1}$ for the normal windshield). The difference manifests itself in the DLF values too - see Fig. 5 for the surface averaged damping loss factor $(\eta)$ values of normal and acoustic windshields besides free and mounted condition. It can be further concluded, that mounting the normal windshield into the chassis significantly increases its damping, while that of the acoustic one changes only moderately.

\subsection{Damping distribution measurements}

Measured DLF distribution confirmed earlier findings and revealed that normal and acoustic windshields could have significantly different damping characteristics at the same frequency-band. In Fig. 6, DLF maps for the normal and acoustic windshields are plotted at two different frequencies. At the lower frequency (Frequency 1) the DLFdistribution is quite similar, while the absolute values are somewhat higher for the acoustic windshield. In contrast, at the higher frequency (Frequency 2) the distribution map is completely different and the measured damping values are significantly higher for the acoustic windshield.

Fig. 7 also shows that the acoustic windshield has significantly higher average damping. However, DLF distribution is similar in a sense that the middle region has higher average damping for both windshields. In turn, the average
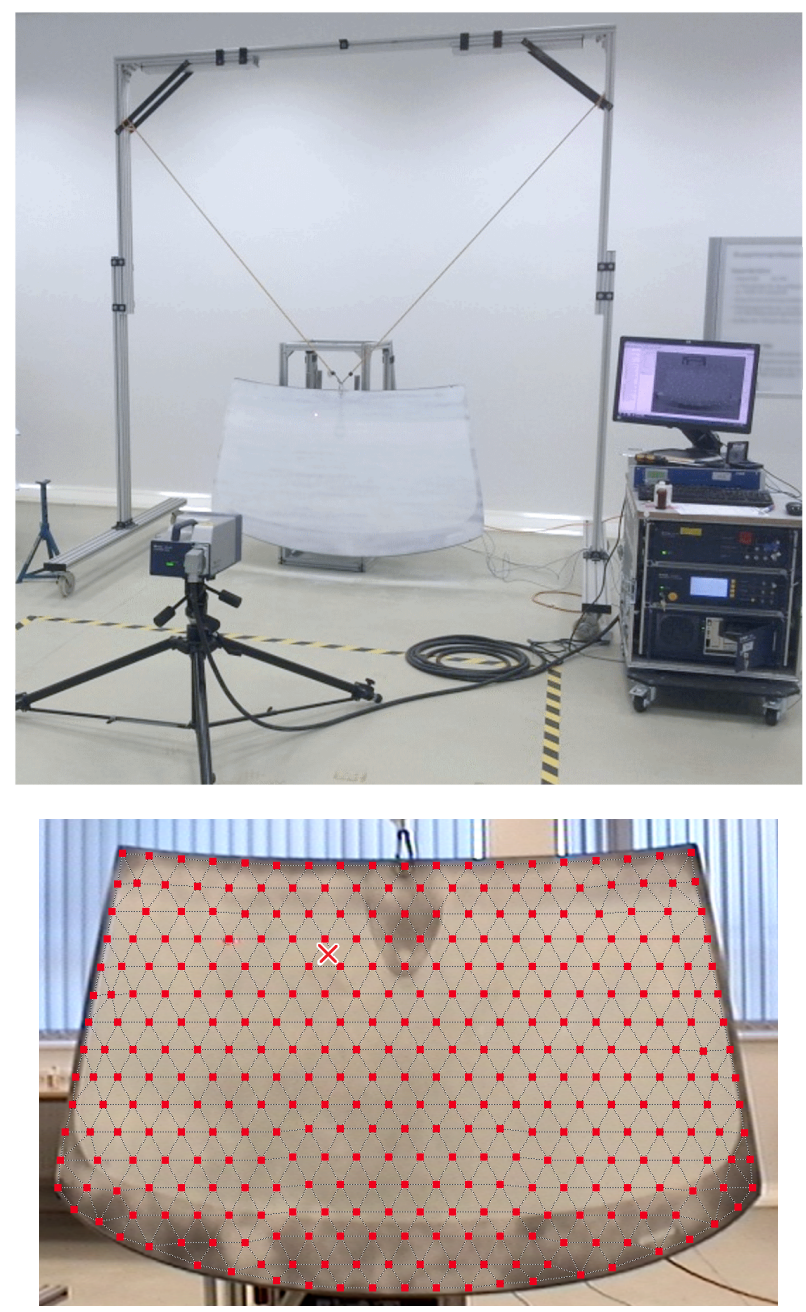

Fig. 3 Vibration shape analysis: measurement setup with laserscanning-vibrometer and evaluation computer (up) and the measured points of the windshield (excitation point marked with $\mathrm{X}$, bottom)

specific deviation (calculated as the frequency-average of the specific deviations from surface-average DLF) indicates that the measured damping of normal windshield has generally higher spatial fluctuation than that of the acoustic one.

\subsection{Vibrometer measurements}

Vibration shapes of the windshields - excited by shaker and measured by laser scanning vibrometer - proved that the position of the excitation point has great impact on the operational vibration shape. In Fig. 8, the vibration shapes of an acoustic windshield can be seen beside 4 different excitation points (stationary excitation) at the same Frequency 2 as indicated in Fig. 6. Based on the symmetry, the last excitation point seems to excite the most effectively the related modal shape.

From the results a crucial conclusion can be extracted: if one compares the bottom-right pictures of Fig. 6 and Fig. 8 


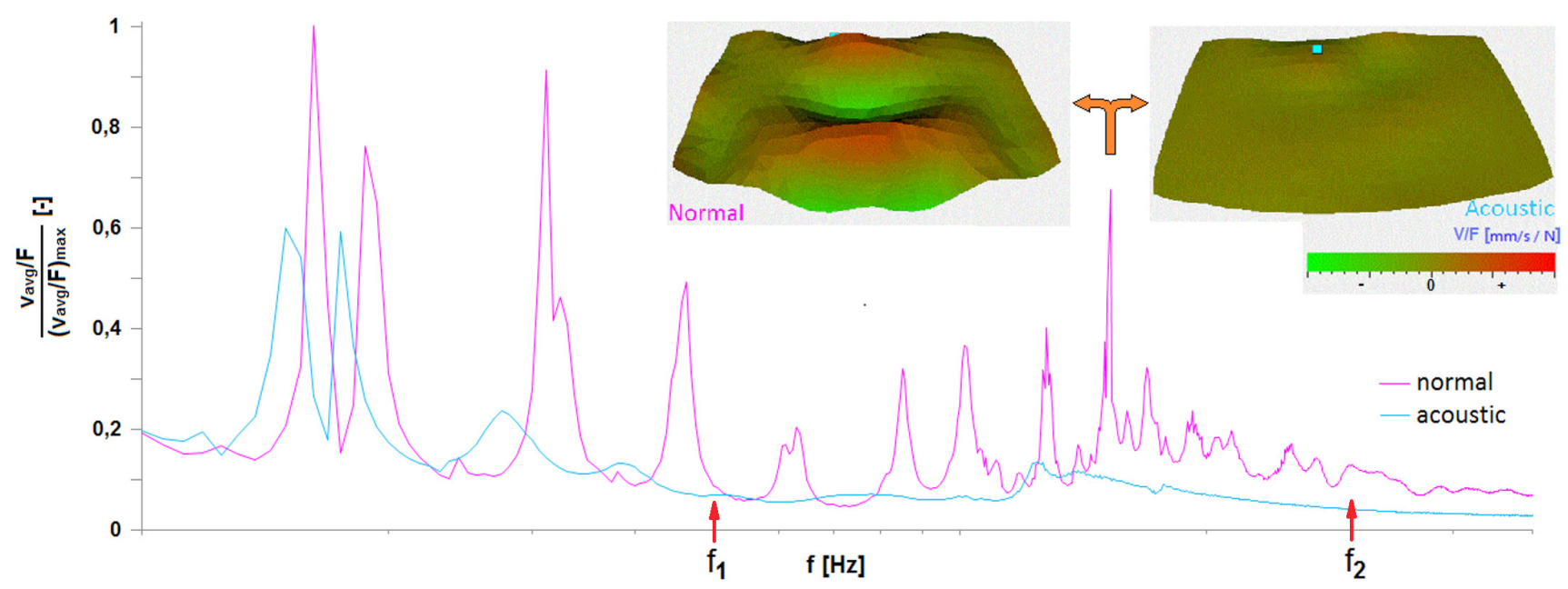

Fig. 4 Normalized transfer functions and maximum surface velocity distribution (at the marked frequency) of normal and acoustic windshields

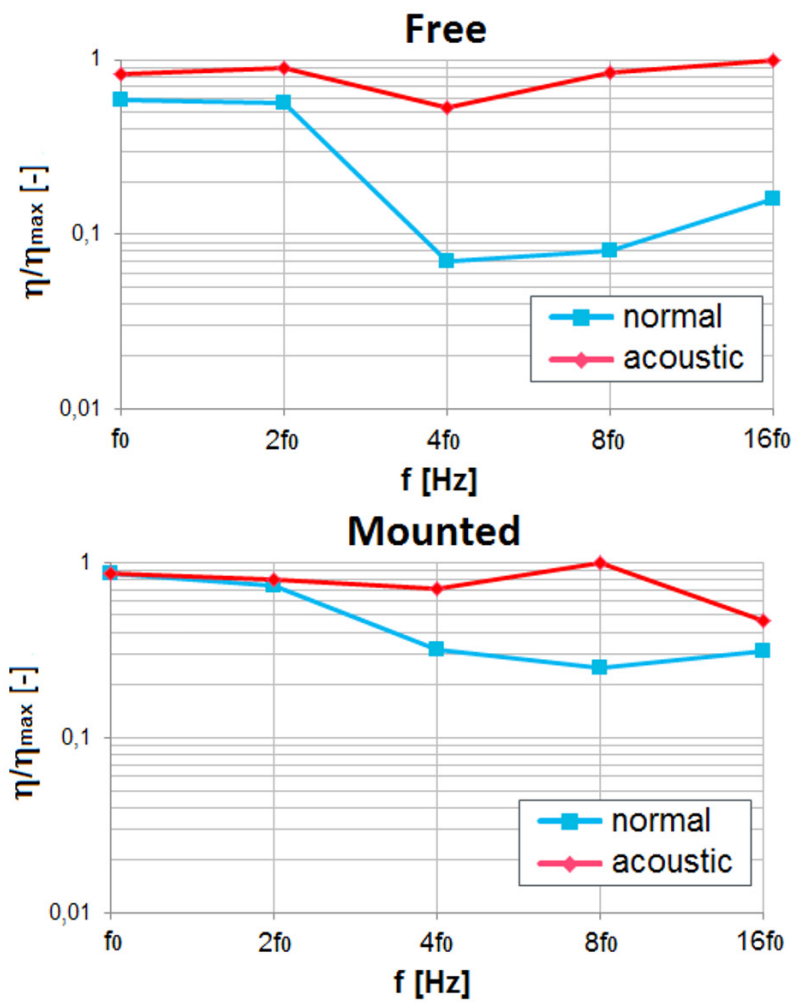

Fig. 5 Surface-average damping loss factor values as a function of frequency for normal and acoustic windshields - measured in free-free state (up) and in mounted state (down)

(i.e. the same windshield at the same frequency but two basically different measurements) the measured vibration shape is reflected quite accurately on the determined DLFdistribution: The regions with high mobility (i.e. high absolute value of vibration velocity response in Fig. 8) are found to show low damping values while higher DLF values were measured for regions with low mobility. This result implies that the modal shapes have dominant impact on the measured DLF values - in other words: the measured damping is highly influenced by geometrical properties and is far from being purely material-characteristic.

\section{Conclusion}

A novel damping measurement method was demonstrated in the paper. Results were checked against data from a highly-efficient scanning vibrometer. It was found, that the measured damping values are strongly related to modal shapes. This appears to be quite useful, since resonance problems can always be linked to a modal vibration (frequency). On the other hand, this means that it might be quite hard to compare different windshields from the point of view of vibration damping, since as geometry is changed the modal shapes and frequencies will change too.

For the future, two research directions are proposed. Firstly, it is suggested to make the damping loss factor measurement more effective by using for example surface-excitation instead of point-based impact measurements. Secondly, develop a measurement method, which can provide reliable and comparable damping values for different windshields independently of the geometry and/ or modal shapes.

\section{Acknowledgement}

The work presented in this article is supported by project EFOP3.6.1-16-2016-00017 ("Internationalization, initiatives to establish a new source of researchers and graduates, and development of knowledge and technological transfer as instruments of intelligent specializations at Széchenyi István University"). 


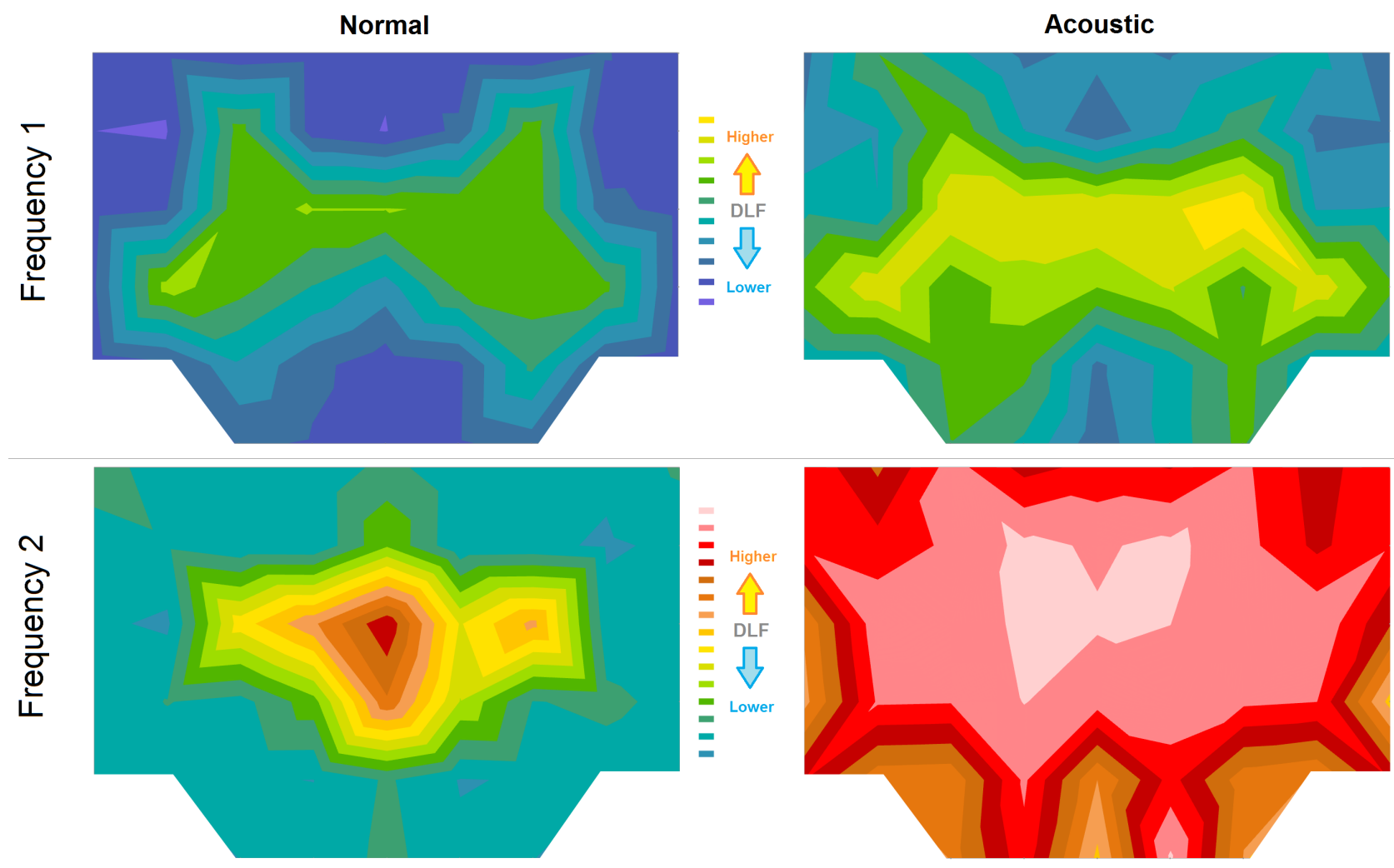

Fig. 6 Damping loss factor distribution at two different frequencies on the normal and acoustic windshields
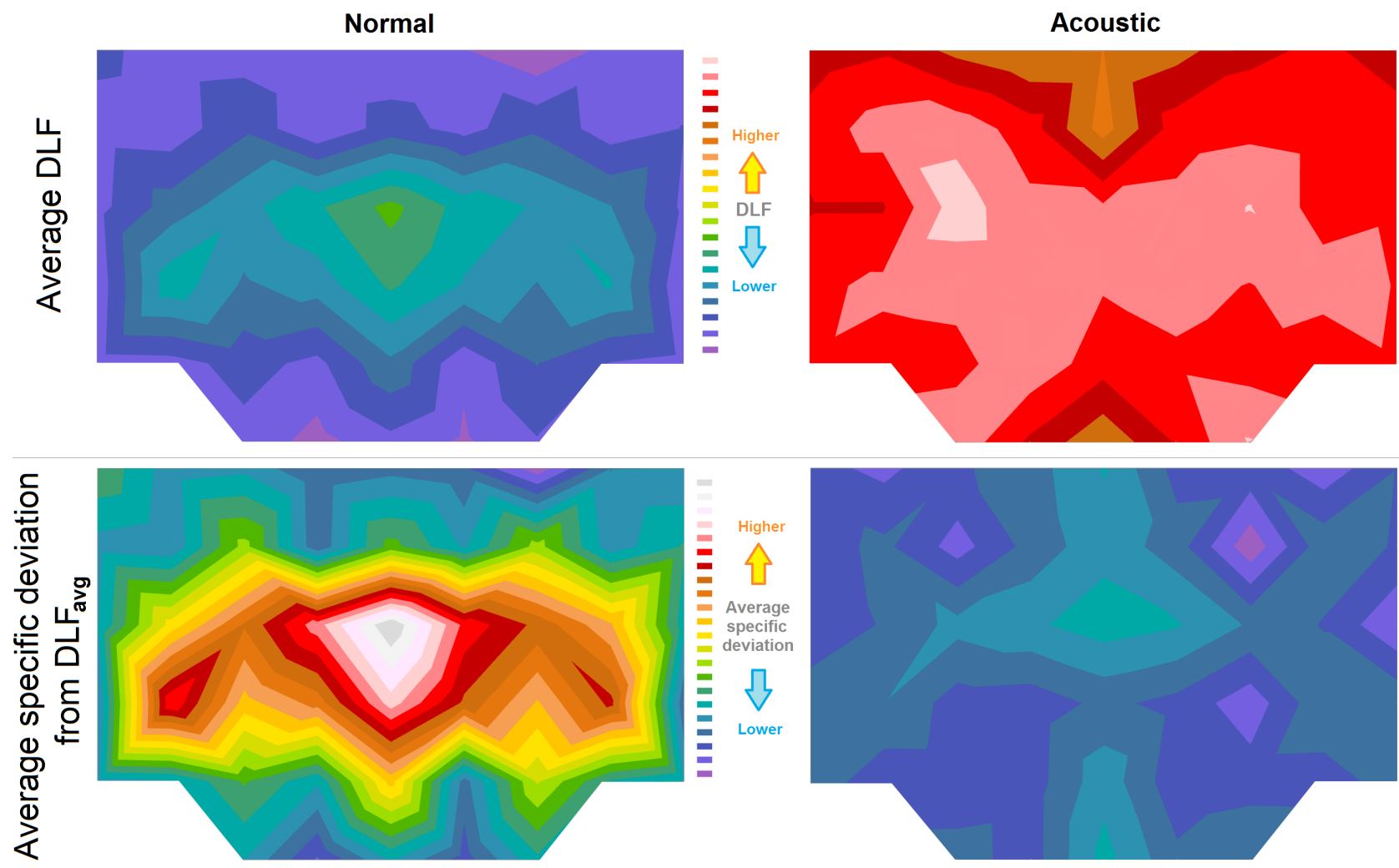

Fig. 7 Frequency-averaged damping loss factor distribution (from the lower audible region) and the frequency-averaged specific deviation from surface-average DLF 


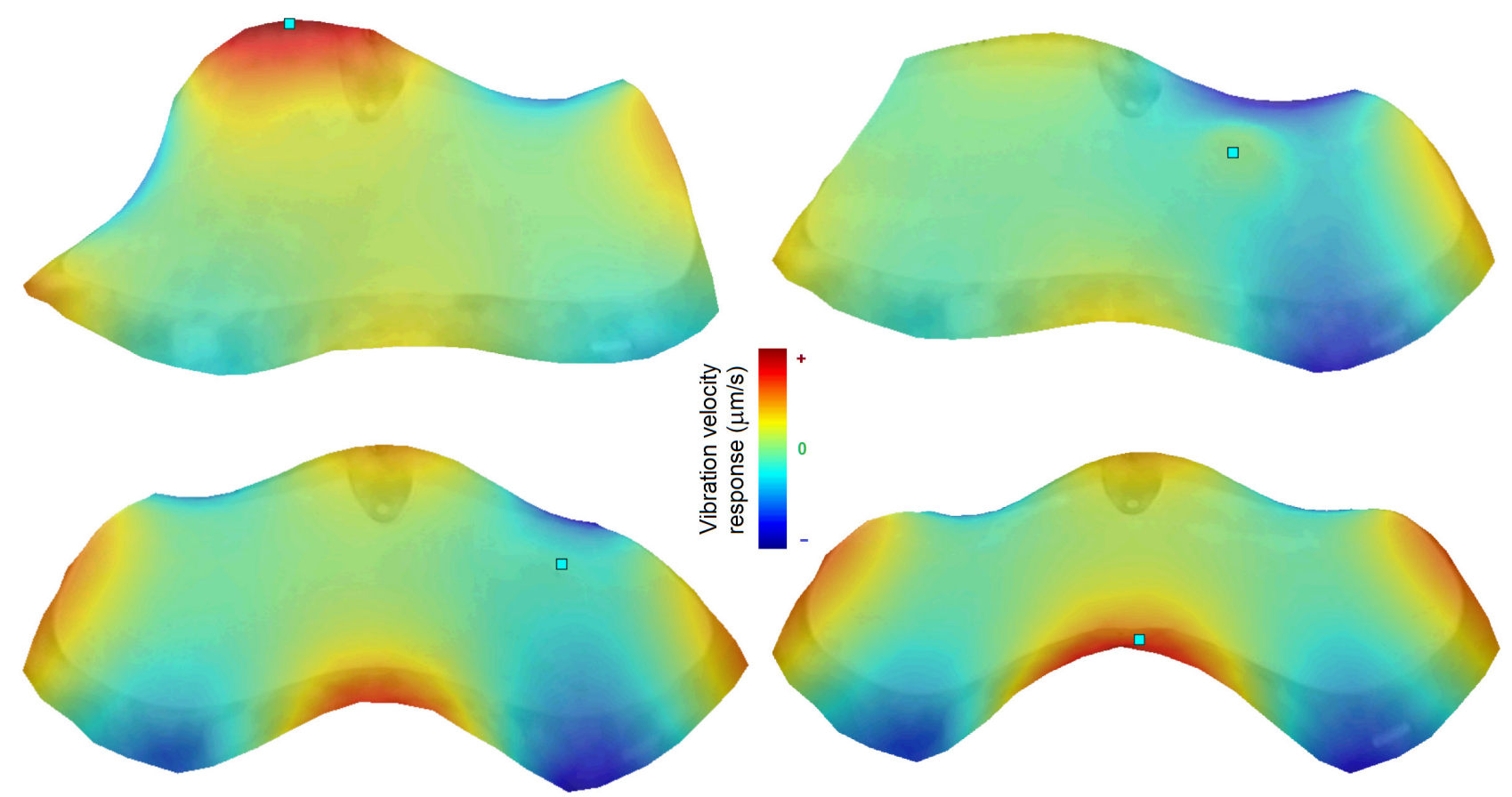

Fig. 8 Measured vibration shapes at the same frequency beside 4 different excitation points

\section{References}

[1] "ASTM E756-98, Standard Test Method for Measuring VibrationDamping Properties of Materials", American Society for Testing and Materials, West Conshohocken, PA, USA, 1998.

https://doi.org/10.1520/E0756-98

[2] Wojtowicki, J.-L., Jaouen, L., Panneton, R. "New approach for the measurement of damping properties of materials using the Oberst beam", Review of Scientific Instruments, 75(8), pp. 2569-2574, 2004. https://doi.org/10.1063/1.1777382

[3] Hinke, L., Pichler, L., Pradlwarter, H. J., Mace, B. R., Waters, T. P. "Modelling of spatial variations in vibration analysis with application to an automotive windshield", Finite Elements in Analysis and Design, 47(1), pp. 55-62, 2011.

https://doi.org/10.1016/j.finel.2010.07.013
[4] Carfagni, M., Lenzi, E., Pierini, M. "The Loss Factor as a Measure of Mechanical Damping", In: 16th, International Modal Analysis Conference, Santa Barbara, CA, USA, 1998, pp. 580-584.

[5] Meier, A. "Die Bedeutung des Verlustfaktors bei der Bestimmung der Schalldämmung im Prüfstand" (The importance of damping loss factor in the determination of sound insulation by test measurement), PhD Theses, Rheinisch-Westfälischen Technischen Hochschule Aachen, Germany, 2000 (in German), [online] Available at: http://sylvester.bth.rwth-aachen.de/dissertationen/2000/23/00_23.pdf [Accessed: 01 September 2017]

[6] Schroeder, M. R. "New Method of Measuring Reverberation Time", Journal of the Acoustical Society of America, 37(3), pp. 409-412, 1965.

https://doi.org/10.1121/1.1909343 